\title{
Resolving the space-time structure of sonoluminescence by intensity interferometry
}

\author{
Claus Slotta and Ulrich Heinz \\ Institut für Theoretische Physik, Universität Regensburg, D-93040 Regensburg, Germany
}

(May 3, 2019)

\begin{abstract}
We analyze the spatial and temporal resolving power of two-photon intensity interferometry for the light emitting source in single bubble sonoluminescence (SBSL). We show that bubble sizes between several $10 \mathrm{~nm}$ and $3 \mu \mathrm{m}$ can be resolved by measuring the transverse correlation function, but that a direct determination of the flash duration via the longitudinal correlation function works only for SBSL pulses which are shorter than 0.1 ps. Larger pulse lengths can be determined indirectly from the intercept of the angular correlator at equal photon frequencies. The dynamics of the bubble is not accessible by two-photon interferometry.

78.60.Mq,43.35.+d,95.75.Kk,25.75.Gz
\end{abstract}

Typeset using REVTEX 


\section{INTRODUCTION}

In single bubble sonoluminescence (SBSL) [1] an air bubble in water is trapped in the velocity node of an acoustical pressure field. Under defined conditions this bubble periodically emits intense, broad-banded flashes of light, synchronous to the driving sound. Measurements of pulse widths have given estimated values from less than $50 \mathrm{ps}$ [1, 2, to more than $250 \mathrm{ps}$ [3], and an analysis of the bubble surface's temporal variation indicates minimal radii of $\sim 0.5 \mu \mathrm{m}$ [1]. A direct measurement of the size of the light emitting region does not exist so far.

The fundamental light generating mechanism is still unknown. Some models [4,5] attribute the light emission to electronic excitations or Bremsstrahlung processes initiated by spherically converging shock fronts, leading essentially to a black-body spectrum. While reproducing the measured spectra quite well they require extraordinarily high temperatures with at least partial ionization of the trapped gas. The peak of this thermal spectrum and its high-energy Boltzmann tail are postulated to be hidden below the absorption edge of water $(\lambda<180 \mathrm{~nm})$ where the photon spectrum cannot be measured. Alternative explanations, invoking collision-induced emission [6] or quantum vacuum radiation [7], operate at more moderate temperatures and, in the latter case, do not exhibit an (invisible) high energy component while still reproducing the spectral shape in the measurable low energy window. Numerical simulations [4] combining the gas dynamics inside a sphere with the oscillations of the bubble surface yield pulse widths of $\sim 1$ ps combined with a radius of $\sim 0.5 \mu \mathrm{m}$ for the emission region. These simulations indeed produce strong shock waves which are seen as causal for the light emission, but the predicted flash duration does not seem to coincide with the experimental data.

Intensity interferometry, based on Bose-Einstein correlations (BEC) between identical bosons, has been discussed as a possibility to clarify the structure and dynamics of the light emitting region [8.9] by directly measuring its size and lifetime. This technique, originally developed to determine the angular size of stars [10], has recently been considerably refined 
in order to extract the spatial and temporal structure of the hot reaction zone created in high energy nuclear collisions [1]]. It has proven to be a valuable tool also in the analysis of dynamical processes in extremely small and short-lived particle sources. Its application to SBSL in the form of two-photon intensity interferometry should be further facilitated by the comparatively large number of photons emitted per pulse and by the absence of many of the complications present in high energy applications, like final state Coulomb and strong interactions among the produced particles and secondary production of particles outside the source by decay of unstable resonances.

If successful, SBSL interferometry may in fact turn out to be a unique testing ground for the method itself since here, contrary to high energy physics where the sources are too small and shortlived to be probed externally, alternative investigation techniques are available which should allow for various cross checks.

In this paper we supplement the suggestion of [8,9] with a general and largely model independent discussion of the method and its application to SBSL, providing a quantitative analysis of its spatial and temporal resolving power in the limited range of experimentally accessible wavelengths. We show that the transverse correlator is sensitive to bubble sizes in the physically interesting domain while direct resolution of the pulse length via the longitudinal correlator is probably not possible with present technology. It can be determined indirectly, however, via the intercept of the transverse correlator at zero opening angle between the detectors. The dynamics of the bubble can, unfortunately, not be resolved interferometrically.

\section{TWO-PHOTON CORRELATION FUNCTION}

The correlation function for two photons with momenta $\boldsymbol{k}_{a}$ and $\boldsymbol{k}_{b}$ is defined as

$$
C\left(\boldsymbol{k}_{a}, \boldsymbol{k}_{b}\right)=\frac{P_{2}\left(\boldsymbol{k}_{a}, \boldsymbol{k}_{b}\right)}{P_{1}\left(\boldsymbol{k}_{a}\right) P_{1}\left(\boldsymbol{k}_{b}\right)},
$$

where $P_{1}(\boldsymbol{k})$ is the inclusive single-photon spectrum and $P_{2}\left(\boldsymbol{k}_{a}, \boldsymbol{k}_{b}\right)$ is the two-photon coin-

cidence spectrum. All photon energies are on-shell, $\omega_{a, b}=\left|\boldsymbol{k}_{a, b}\right|$. In the following we assume 
that the photons are emitted completely incoherently and that the source is spherically symmetric. While the latter is strongly suggested by the extreme stability of the oscillating bubble, some phase coherence among the emitted photons can presently not be excluded. Ideally one would test this by measuring the (true) intercept of the two-photon correlator (四) at $\boldsymbol{q}=\boldsymbol{k}_{a}-\boldsymbol{k}_{b}=0$ [9]. As we will show such a measurement is difficult, due to the limited frequency resolution of present photon detectors; in the long run one might therefore contemplate a comparison of 2- and 3-photon interferometry data to settle the issue of partial coherence [12].

For chaotic (incoherent) sources the single-photon spectrum $P_{1}(\boldsymbol{k})$ and the correlator $C\left(\boldsymbol{k}_{a}, \boldsymbol{k}_{b}\right)$ can be expressed in terms of the single-photon Wigner phase-space density $S(x ; K)$ of the emitting source 13 15]:

$$
\begin{aligned}
P_{1}(\boldsymbol{k}) & =\int d^{4} x S(x ; \boldsymbol{k}, \omega) \\
C\left(\boldsymbol{k}_{a}, \boldsymbol{k}_{b}\right) & =1+\frac{1}{2} \frac{\left|\int d^{4} x S(x ; K) e^{\mathrm{i} q \cdot x}\right|^{2}}{\int d^{4} x S\left(x ; \boldsymbol{k}_{a}, \omega_{a}\right) \int d^{4} y S\left(y ; \boldsymbol{k}_{b}, \omega_{b}\right)} \\
& \approx 1+\frac{1}{2}\left|\frac{\int d^{4} x S(x ; \boldsymbol{K}, E) e^{\mathrm{i} q \cdot x}}{\int d^{4} x S(x ; \boldsymbol{K}, E)}\right|^{2}
\end{aligned}
$$

Here $K=\left(\omega_{a}+\omega_{b}, \boldsymbol{k}_{a}+\boldsymbol{k}_{b}\right) / 2$ and $q=\left(\omega_{a}-\omega_{b}, \boldsymbol{k}_{a}-\boldsymbol{k}_{b}\right)$. The second equation in (3) is an approximation in that the single-photon spectra in the denominator have been evaluated at the average momentum $\boldsymbol{K}$ rather than at $\boldsymbol{k}_{a}$ and $\boldsymbol{k}_{b}$, and in both the numerator and denominator the correct energy variables $\left(\omega_{a}, \omega_{b}\right.$, and $K_{0}=\left(\omega_{a}+\omega_{b}\right) / 2$, respectively) have been replaced by the on-shell energy corresponding to $\boldsymbol{K}, E=\left|\boldsymbol{k}_{a}+\boldsymbol{k}_{b}\right| / 2$. This approximation makes the following discussion more transparent, but can be systematically corrected for [16] (see below). The factor $\frac{1}{2}$ in front of the second term in the correlator (3) takes into account [17] that only photons with equal helicity states are affected by Bose symmetrization.

Since the measured photons are on-shell and thus the Fourier transform in (3) is not invertible, the space-time structure of $S(x ; K)$ cannot be uniquely reconstructed. Still, valuable information on the space-time structure of the source can be extracted from the 
measured correlation function in terms of the second central space-time moments of $S(x ; K)$ [16,18,[1]. In the context of SBSL this will be discussed next.

\section{GEOMETRICAL ANALYSIS}

Detailed investigations (for a recent overview see [11]) have shown that the essential features of the correlator (3) can be captured by replacing the $x$-dependence of the emission function $S(x ; K)$ by a Gaussian with the same center and width. This is even more true for SBSL applications than in high energy particle physics since here resonance decay effects which can invalidate this Gaussian approximation are absent. Inserting a Gaussian ansatz for $S(x ; K)$ into (3) yields a correlator which is Gaussian in the relative momentum $q$. Due to the spherical symmetry of the problem there is only one distinguished direction which is defined by the photon pair momentum $\boldsymbol{K}$. We therefore use a Cartesian coordinate system in which $\boldsymbol{K}=(E, 0,0)$ (i.e. all $K$-dependence can be expressed through the energy $E$ ) and $\boldsymbol{q}=\left(q_{\|}, q_{\perp}, 0\right)$. We also have $q_{0}=\omega_{a}-\omega_{b}=\frac{E}{\omega_{a}+\omega_{b}} q_{\|} \approx q_{\|}$. Following the techniques developed in [16,18] it is then easily seen that the most general form of the correlator reads

$$
C(\boldsymbol{q}, E) \approx 1+\frac{1}{2} e^{-q_{\perp}^{2}\left\langle x_{\perp}^{2}\right\rangle(E)-q_{\|}^{2}\left\langle\left(\tilde{x}_{\|}-c \tilde{t}\right)^{2}\right\rangle(E)},
$$

where the angular brackets denote averages taken with the source function,

$$
\langle f(x)\rangle(E)=\frac{\int d^{4} x f(x) S(x ; E)}{\int d^{4} x S(x ; E)},
$$

and tilde superscripts indicate center-corrected coordinates, $\tilde{x}_{i}=x_{i}-\left\langle x_{i}\right\rangle(E)$. (Note that $\left\langle x_{\perp}\right\rangle(E)=0$ due to spherical symmetry.) Eq. (四) tells us that by measuring, at fixed $E$, the correlator as a function of $q_{\perp}$ and $q_{\|}$, respectively, we can determine the spatial variance $\left\langle x_{\perp}^{2}\right\rangle$ and the mixed variance $\left\langle\left(c \tilde{t}-\tilde{x}_{\|}\right)^{2}\right\rangle$, respectively, of the effective source of photons with

energy $E$. In principle, for different $E$ the effective source can have different such "sizes" or "HBT radii".

In relativistic heavy ion collisions the $K$-dependence (here: $E$-dependence) of the spacetime variances plays an important role as a signature for collective expansion of the emitting 
source [11]. This is most easily seen in the context of a hydrodynamically expanding, locally thermalized source whose momentum dependence is dominated by a boosted Boltzmann distribution $\sim \exp [-K \cdot u(x) / T(x)]$ where $u(x)$ is the collective expansion 4-velocity profile. This factor generates correlations between the momentum $K$ and the position $x$ in the emitter which in turn cause a $K$-dependence of the HBT radii. The strength of these correlations can be estimated by writing

$$
S(x ; E) \sim s(K \cdot u(r, t) / T) \approx s(E / T)(1+\mathcal{O}(v / c))
$$

Since the expansion velocity $v(r, t)$ of the bubble in SBSL is limited by the shock velocity in the compressed bubble gas and thus below about $3 \times 10^{4} \mathrm{~m} / \mathrm{s}$ [4] (i.e. $v / c<10^{-4}$ ), the nonrelativistic estimate (6) is reliable and the $x-K$ correlations induced by the collective dynamics of the bubble are weak. This is different for pion interferometry in heavy ion collisions: there the collective velocities are of the order of the light velocity, causing strong $x$ - $K$ correlations and an appreciable $K$-dependence of the correlator which can be used as a diagnostic tool [11]. In SBSL interferometry, on the other hand, the $E$-dependence of the HBT radii resulting from the weak $x-K$ correlations is so small that it can be neglected in the measurable E-range (see below). This is unfortunate since it means that SBSL interferometry will not give any direct access to the collective dynamics of the bubble during light emission [19]. On the other hand, it simplifies the theoretical description because we can neglect the $E$-dependence of the HBT radii and also drop the cross term in the longitudinal HBT radius:

$$
\left\langle\left(\tilde{x}_{\|}-c \tilde{t}\right)^{2}\right\rangle \approx\left\langle x_{\|}^{2}\right\rangle+c^{2}\left\langle\tilde{t}^{2}\right\rangle
$$

This is true because both the displacement $\left\langle x_{\|}\right\rangle$of the source center in $\boldsymbol{K}$-direction and the cross term $\left\langle x_{\|} t\right\rangle$ are also generated by the collective expansion [16] and thus here expected to be about 4 orders of magnitude smaller than the diagonal terms $\left\langle x_{\|}^{2}\right\rangle$ and $\left\langle\tilde{t}^{2}\right\rangle$.

The corrections resulting from the approximation (3) can now be systematically included following the discussion in [16]. Writing up to second order in $q$ 


$$
\frac{\left(\omega_{a}+\omega_{b}\right)^{2}}{4} \approx E^{2}+\frac{1}{4}\left(\boldsymbol{q}^{2}-\frac{(\boldsymbol{K} \cdot \boldsymbol{q})^{2}}{E^{2}}\right)
$$

one derives to quadratic accuracy

$$
\int d^{4} x S\left(x ; \boldsymbol{K}, K_{0}\right) e^{\mathrm{i} q \cdot x} \approx e^{\Delta R_{\perp}^{2} q_{\perp}^{2}} \int d^{4} x S(x ; \boldsymbol{K}) e^{\mathrm{i} q \cdot x}
$$

with

$$
\Delta R_{\perp}^{2}(E)=\left(\frac{1}{8 E} \frac{\mathrm{d}}{\mathrm{d} E} \ln P_{1}(E)\right) .
$$

The single particle distribution $P_{1}\left(\omega_{a}\right)$ may be similarly approximated by

$$
P_{1}\left(\omega_{a}\right) \approx\left(1+\left(\frac{q_{\perp}^{2}}{8 E}+\frac{q_{\|}}{2}\right) \frac{\mathrm{d}}{\mathrm{d} E}+\frac{q_{\|}^{2}}{8} \frac{\mathrm{d}^{2}}{\mathrm{~d} E^{2}}\right) P_{1}(E)
$$

For $P_{1}\left(\omega_{b}\right)$ one obtains the same expression with the opposite sign for the term linear in $q_{\|}$. For the denominator in (3) one thus finds to quadratic order

$$
P_{1}\left(\omega_{a}\right) P_{1}\left(\omega_{b}\right) \approx e^{2 \Delta R_{\perp}^{2} q_{\perp}^{2}+\Delta R_{\|}^{2} q_{\|}^{2}} P_{1}^{2}(E)
$$

with

$$
\Delta R_{\|}^{2}(E)=\frac{1}{4} \frac{\mathrm{d}^{2}}{\mathrm{~d} E^{2}} \ln P_{1}(E)
$$

Hence the corrected Gaussian expression (4) for the correlator reads

$$
\begin{aligned}
C\left(q_{\perp}, q_{\|}, E\right) & \approx 1+\frac{1}{2} e^{-R_{\perp}^{2} q_{\perp}^{2}-R_{\|}^{2} q_{\|}^{2}}, \\
R_{\perp}^{2} & =\left\langle x_{\perp}^{2}\right\rangle+\Delta R_{\perp}^{2}, \\
R_{\|}^{2} & =\left\langle x_{\|}^{2}\right\rangle+c^{2}\left\langle\tilde{t}^{2}\right\rangle+\Delta R_{\|}^{2} .
\end{aligned}
$$

Note that the corrections $\Delta R_{\perp}^{2}$ and $\Delta R_{\|}^{2}$ are proportional to the slope and curvature of the logarithmic intensity spectrum, respectively, and are therefore directly accessible from single-photon measurements. Both $\Delta R_{\perp}$ and $\Delta R_{\|}$turn out to be at most several $10 \mathrm{~nm}$.

The experimental realization of the correlation measurement (as proposed by Trentalange and Pandey [8]) consists of two photo-multipliers focussing on the sonoluminating bubble 
at a relative angle $\phi$. The required momentum resolution is achieved by suitably chosen apertures and pre-detector band-pass filters. The signal detected in one multiplier during one flash, proportional to the incident number of photons, is correlated with the output of the second device during the same flash and sampled over a sufficient number of bubble oscillations for statistics.

This experimental setup suggests the use of $q_{0}=\omega_{a}-\omega_{b}$ and $\phi$ instead of the variables $q_{\perp}$ and $q_{\|}$. They are related via

$$
\begin{aligned}
& q_{\perp}^{2}=\left(4 E^{2}+\frac{q_{0}^{4}}{4 E^{2}}-2 q_{0}^{2}\right) \tan ^{2} \frac{1}{2} \phi \\
& q_{\|}^{2}=q_{0}^{2}+\left(q_{0}^{2}-\frac{q_{0}^{4}}{4 E^{2}}\right) \tan ^{2} \frac{1}{2} \phi .
\end{aligned}
$$

$R_{\perp}$ and $R_{\|}$can thus be isolated by fixing the average photon energy $E$ and scanning the correlator either as a function of the opening angle at equal photon energies $\left(q_{0}=0\right.$, "transverse correlator"), or as a function of the energy difference $q_{0}$ at zero opening angle $(\phi=0$, "longitudinal correlator"):

$$
\begin{aligned}
& C\left(q_{0}=0, \phi, E\right) \approx 1+\frac{1}{2} \exp \left(-R_{\perp}^{2} 4 E^{2} \tan ^{2} \frac{1}{2} \phi\right) \\
& C\left(q_{0}, \phi=0, E\right) \approx 1+\frac{1}{2} \exp \left(-R_{\|}^{2} q_{0}^{2}\right) .
\end{aligned}
$$

\section{RESOLVING POWER}

We will now proceed towards a quantitative estimate of the resolving power of such correlation measurements. We begin by noting that our approximations break down if the first terms on the right hand sides of $(13 \mathrm{~b}, \mathrm{c})$ become smaller than the corrections from the second terms. This turns out not to be the limiting factor, though, because similar lower limits for the HBT radii result from the fact that the opacity of water prohibits measurements at wavelengths in the ultraviolet below $180 \mathrm{~nm}$, and low yields make the measurement difficult in the infrared $(\omega \lesssim 1.5 \mathrm{eV})$. Practical measurements are only possible in the "transparency window" $1.5 \mathrm{eV} \lesssim \omega \lesssim 6 \mathrm{eV}(210 \mathrm{~nm} \lesssim \lambda \lesssim 830 \mathrm{~nm})$. As we will see 
this limits, at fixed $E$, the opening angle $\phi$ and the energy difference $q_{0}$ which means that the correlator (15) can only be measured over a restricted interval of the control variables. If the correlator does not fall off appreciably over the accessible range, the HBT radius parameter cannot be accurately determined. This gives lower limits for $R_{\perp}$ and $R_{\|}$of several $10 \mathrm{~nm}$, i.e. of the same order of magnitude as the upper limits for the correction terms $\Delta R_{\|}, \Delta R_{\perp}$.

After these general remarks let us enter a more detailed discussion, beginning with $R_{\perp}$. In Fig. 1 we have plotted the correlator (15a) at fixed $E=3 \mathrm{eV}$ and $q_{0}=0$ as a function of the opening angle $\phi$. One sees that for $R_{\perp}=10 \mathrm{~nm}$ the correlator falls off only by about $20 \%$ over the measurable range, rendering the determination of $R_{\perp}$ difficult. According to Fig. 1, good measurements of $R_{\perp}$ are possible for $10 \mathrm{~nm}<R_{\perp}<3 \mu \mathrm{m}$. For $R_{\perp}>3 \mu \mathrm{m}$ angular resolution becomes a problem: the correlator falls off so rapidly that opening angles between the two detectors and angular apertures of each detector below $1^{\circ}$ are required to resolve the correlation function. This obviously cuts down on event statistics. However, $R_{\perp}>3 \mu \mathrm{m}$ implies a source with transverse size $\sqrt{\left\langle x_{\perp}^{2}\right\rangle} \gtrsim 3 \mu \mathrm{m}$, see Eq. (13b). Since it is known that at the point of light emission the source is smaller than this (values below $1 \mu \mathrm{m}$ are quoted in [1]), angular resolution of the measurement does not appear to be a crucial limiting factor.

One should note, however, that $q_{0}=0$ as indicated in Fig. 1 implies ideal energy resolution of the photon detector. We will see shortly that the finite energy resolution in real life modifies significantly the optimistic picture suggested by Fig. 1.

Let us now turn to a discussion of $R_{\|}$. It is easy to see that if the light emitting source has a radius below $1 \mu \mathrm{m}$ (i.e. $\sqrt{\left\langle x_{\|}^{2}\right\rangle} \lesssim 1 \mu \mathrm{m}$ ) then the r.h.s. of (13c) is dominated by the duration of the light flash $\delta \tau=\sqrt{\left\langle\tilde{t}^{2}\right\rangle}$ as soon as $\delta \tau$ becomes larger than about 3 femtoseconds (which corresponds to $c \delta \tau=1 \mu \mathrm{m}$ ). Since typical SBSL pulse durations discussed in the literature [1] 20 are much longer we can for the following discussion neglect in (13c) the geometric contribution as well as $\Delta R_{\perp}$ and write $R_{\|} \approx c \delta \tau$.

Fig. 2 shows that SBSL pulses which last longer than 1 ps can only be resolved if the photon detector has an energy resolution well below $1 \mathrm{meV}(!)$. This implies a relative band 
width $\delta \lambda / \lambda \lesssim 10^{-4}$. As we will see in a moment, it is not sufficient that the filter-to-filter distance between the two detectors is known with this accuracy; the band width of each filter individually must satisfy this constraint.

Commercially available filters in the visible region around $400 \mathrm{~nm}$ have band widths $\delta \lambda \gtrsim 1 \mathrm{~nm}$, corresponding to $\delta \lambda / \lambda \gtrsim 25 \times 10^{-4}$. For $\delta \lambda=10 \mathrm{~nm}$ the authors of [8] quoted coincidence rates of 200-300 counts/s at a bubble-detector distance of $200 \mathrm{~mm}$. For smaller $\delta \lambda$ the coincidence rate drops essentially like $(\delta \lambda)^{2}$.

Too large values of $\delta \lambda / \lambda$ imply that the correlator in Fig. 2 is averaged over a range $q_{0}$ which is much larger than the region over which the correlator drops back to 1 . This implies not only that the longitudinal correlation function $C\left(q_{0}\right)$ cannot be resolved, but also that the transverse correlation function $C(\phi)$, being averaged over a wide $q_{0}$-range, is strongly diluted. As a consequence $C(\phi)$ will not intercept the vertical $(\phi=0)$-axis at the ideal value $1+\frac{1}{2}=\frac{3}{2}$, but at a much lower value. The effective intercept will be the smaller the larger the band width of the photon detector.

It is not difficult to calculate the effective intercept value as a function of the ratio between the filter band width and the flash duration. Let us assume filters with a Gaussian frequency profile

$$
f_{\bar{\omega}, \delta \omega}(\omega)=\frac{1}{\sqrt{2 \pi(\delta \omega)^{2}}} \exp \left[-\frac{(\omega-\bar{\omega})^{2}}{2(\delta \omega)^{2}}\right] .
$$

One easily checks that

$$
f_{\omega_{a}, \delta \omega}\left(\omega_{1}\right) f_{\omega_{b}, \delta \omega}\left(\omega_{2}\right)=f_{K_{0}, \delta \omega^{\prime}}(\omega) f_{q 0,2 \delta \omega^{\prime}}(\Delta \omega)
$$

where $\delta \omega^{\prime}=\delta \omega / \sqrt{2}, \omega=\left(\omega_{1}+\omega_{2}\right) / 2, \Delta \omega=\omega_{1}-\omega_{2}$, and $K_{0}=\left(\omega_{a}+\omega_{b}\right) / 2, q_{0}=\omega_{a}-\omega_{b}$ as before. Neglecting $x$ - $K$ correlations in the source as discussed above we can assume that $S(x ; K)$ factorizes, $S(x ; E) \approx X(x) \cdot s(E)$, where $X(x)$ is normalized, $\int d^{4} x X(x)=1$. For the product of single particle spectra in the denominator of Eq. (3) we thus obtain

$$
P_{1}\left(\boldsymbol{k}_{a}\right) P_{1}\left(\boldsymbol{k}_{b}\right)=
$$




$$
\begin{gathered}
\int d \omega_{1} d \omega_{2} f_{\omega_{a}, \delta \omega}\left(\omega_{1}\right) f_{\omega_{b}, \delta \omega}\left(\omega_{2}\right) s\left(\omega_{1}\right) s\left(\omega_{2}\right)= \\
\int d \omega f_{K_{0}, \delta \omega^{\prime}}(\omega) \int d(\Delta \omega) f_{q_{0}, 2 \delta \omega^{\prime}}(\Delta \omega) \\
\times s\left(\omega+\frac{1}{2} \Delta \omega\right) s\left(\omega-\frac{1}{2} \Delta \omega\right),
\end{gathered}
$$

while the numerator is similarly found to be

$$
\begin{aligned}
& P_{2}\left(\boldsymbol{k}_{a}, \boldsymbol{k}_{b}\right)-P_{1}\left(\boldsymbol{k}_{a}\right) P_{1}\left(\boldsymbol{k}_{b}\right)= \\
& \frac{1}{2} \int d \omega(s(\omega))^{2} f_{K_{0}, \delta \omega^{\prime}}(\omega) \int d^{4} x d^{4} y X(x) X(y) \\
& \times e^{-i \omega(\boldsymbol{x}-\boldsymbol{y}) \cdot\left(\boldsymbol{e}_{a}-\boldsymbol{e}_{b}\right)} \int d(\Delta \omega) f_{q_{0}, 2 \delta \omega^{\prime}}(\Delta \omega) \\
& \times e^{i \Delta \omega\left[\left(x^{0}-y^{0}\right)-\frac{1}{2}(\boldsymbol{x}-\boldsymbol{y}) \cdot\left(\boldsymbol{e}_{a}+\boldsymbol{e}_{b}\right)\right]}
\end{aligned}
$$

where $\boldsymbol{e}_{a, b}$ are unit vectors in direction of $\boldsymbol{k}_{a, b}$. Since the filter band width $\delta \omega$ is narrow, the single particle spectrum $s(E)$ can be taken constant inside the filter gap. This so-called "smoothness approximation" allows to perform the integration over $\Delta \omega$ in (18):

$$
P_{1}\left(\boldsymbol{k}_{a}\right) P_{1}\left(\boldsymbol{k}_{b}\right) \approx \int d \omega(s(\omega))^{2} f_{K_{0}, \delta \omega^{\prime}}(\omega)
$$

To obtain the effective intercept we divide (19) by (18) and set $k_{a}=k_{b}=K$, i.e. $q_{0}=\phi=0$ and $\boldsymbol{e}_{a}=\boldsymbol{e}_{b}$. Then the $\omega$-integration in Eq. (19) factorizes, and the first factor on the r.h.s. of Eq. (19) cancels against (20). The second factor can be easily evaluated in the Gaussian approximation where we replace the space-time factor $X(x)$ by a Gaussian with the same rms widths. We find

$$
\begin{aligned}
C(q=0) & =1+\frac{1}{2} \frac{1}{\sqrt{1+4(\delta \omega)^{2}\left(\left\langle x_{\|}^{2}\right\rangle+c^{2}\left\langle\tilde{t}^{2}\right\rangle\right) /(\hbar c)^{2}}} \\
& =1+\frac{1}{2} \frac{1}{\sqrt{1+4(\delta \omega)^{2} R_{\|}^{2} /(\hbar c)^{2}}} .
\end{aligned}
$$

The dependence of this effective intercept on $R_{\|}$(which in the limit considered here is approximately equal to the flash duration $c \delta \tau)$ is plotted in Fig. 3 for a fixed filter band width $\delta \lambda=1 \mathrm{~nm}$ at an average photon energy $E=3 \mathrm{eV}$ (corresponding to $\lambda=413 \mathrm{~nm}$ ). For this case $\delta \omega R_{\|} /(\hbar c)=1$ corresponds to a flash duration $\delta \tau=88 \mathrm{fs} \approx 0.1 \mathrm{ps}$. One 
sees that the effective intercept is unity for $\delta \tau \ll 0.1 \mathrm{ps}$; this is the domain where the given filter band width allows to resolve the flash duration by measuring the longitudinal correlation function (15b). For $\delta \tau \gg 0.1 \mathrm{ps}$ the effective intercept decreases linearly with the pulse length, $C(0)-1 \sim 1 / \delta \tau$. Thus, assuming completely incoherent photon emission

and $c \delta \tau \gg \sqrt{\left\langle x_{\|}^{2}\right\rangle}, \delta \tau$ can be determined from the effective intercept of the transverse twophoton correlator (15a) even if the longitudinal correlator (15b) does not show any structure for $\delta$-values outside the experimental resolution (filter band width) $\delta \omega$.

It is worth pointing out that according to this analysis it is not necessarily advisable to strive for increasingly better filter resolution $\delta \omega$ resp. $\delta \lambda$. As discussed above, increasing the band width enhances the coincidence rate quadratically while the effective intercept value decreases only linearly. In leading order the effect of $\delta \lambda$ on the experimental error bar of $\delta \tau$ thus cancels. Of course, determining $\delta \tau$ via the effective intercept of the correlator is a somewhat roundabout procedure which depends in a crucial way on the assumed chaoticity of the source; eventually one would like to achieve a genuine lifetime determination by measuring the longitudinal correlator (15b) with appropriate frequency resolution.

\section{SUMMARY}

We have shown that two-photon intensity interferometry can be applied to study the size and lifetime of the light-emitting region in single-bubble sonoluminescence. A measurement of the transverse correlation function in the experimentally accessible frequency range provides sensitivity to sizes between several $10 \mathrm{~nm}$ and a few $\mu \mathrm{m}$ for the active bubble region. Present technological limitations on the frequency resolution limit a direct measurement of the flash duration via the longitudinal correlation function to pulse lengths below 0.1 ps. We showed, however, that for chaotic emitters with longer flashes the pulse duration can be determined indirectly via the intercept of the transverse correlation function as a function of frequency resolution. The dynamics of the bubble during light emission is not accessible by two-photon interferometry, due to the much too small expansion velocities. 


\section{ACKNOWLEDGMENTS}

U.H. would like to thank B. Svetitsky for interesting discussions which motivated this work. He also gratefully acknowledges clarifying conversations with A. Chodos, Y. Hama,

T. Hemmick, G. Kunde, B. Lasiuk, S. Padula, and S. Trentalange. This work was supported by DFG, BMBF and GSI. 


\section{REFERENCES}

[1] B.P. Barber et al., Phys. Rep. 281, 65 (1997), and references therein.

[2] M.J. Moran et al., Nucl. Instrum. Meth. B 96, 651 (1995).

[3] B. Gompf, R. Günther, G. Nick, R. Pecha, and W. Eisenmenger, Phys. Rev. Lett. 79, 1405 (1997).

[4] C.C. Wu and P.H. Roberts, Phys. Rev. Lett. 70, 3424 (1993); Proc. Roy. Soc. London A 445, $323(1994)$.

[5] R. Hiller, S.J. Putterman, and B.P. Barber, Phys. Rev. Lett. 69, 1182 (1992).

[6] L. Frommhold and A.A. Atchley, Phys. Rev. Lett. 73, 2883 (1994).

[7] C. Eberlein, Phys. Rev. A 53, 2772 (1996); A. Chodos, Los Alamos e-print archive hep-ph/9604368.

[8] S. Trentalange and S.U. Pandey, J. Acoust. Soc. Am. 99, 2439 (1996).

[9] Y. Hama, T. Kodama, and S.S. Padula, Phys. Rev. A 56, 2233 (1997).

[10] R. Hanbury Brown and R.Q. Twiss, Phil. Mag., Ser. 7, Vol. 45, 663 (1954).

[11] U. Heinz, in Correlations and Clustering Phenomena in Subatomic Physics, M.N. Harakeh, J.H. Koch, and O. Scholten, eds., NATO ASI Series B 359, p. 137 (Plenum, New York, 1997).

[12] Q.H. Zhang and U. Heinz, Phys. Rev. C 56, 426 (1997).

[13] E. Shuryak, Phys. Lett. B 44, 387 (1973); Sov. J. Nucl. Phys. 18, 667 (1974).

[14] S. Pratt, T. Csörgő, and J. Zimányi, Phys. Rev. C 42, 2646 (1990).

[15] S. Chapman and U. Heinz, Phys. Lett. B 340, 250 (1994).

[16] S. Chapman, P. Scotto, and U. Heinz, Heavy Ion Phys. 1, 1 (1995). 
[17] C. Slotta and U. Heinz, Phys. Lett. B 391, 469 (1997).

[18] S. Chapman, J.R. Nix, and U. Heinz, Phys. Rev. C 52, 2694 (1995).

[19] In the quantum vacuum radiation scenario of Ref. [7] time profiles $R(t)$ for the bubble radius were used which lead to much larger radial velocities which might indeed be measurable via an E-dependence of the HBT radii. As pointed out in [7] these large radial velocities are not really necessary for the scenario to work because what really matters for the light generation process is the $4^{\text {th }}$ time derivative of the radial velocity at the turnaround point. Still, establishing the (approximate) E-independence of the HBT radii conjectured by us would put significant empirical constraints on dynamical scenarios like the one discussed in [7]. 


\section{FIGURES}

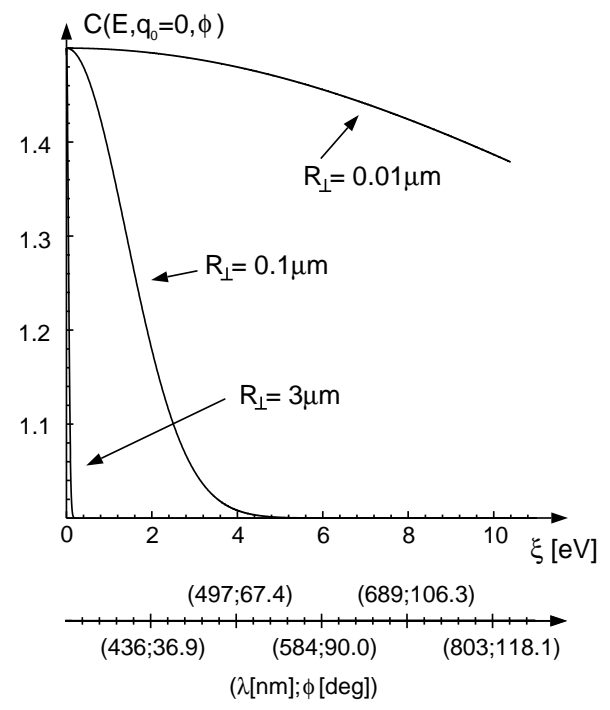

FIG. 1. The correlation function $C\left(E=3 \mathrm{eV}, q_{0}=0, \phi\right)$ as a function of $\xi=2 E \tan \frac{1}{2} \phi$ for various values of $R_{\perp}$. The domain above $\xi \approx 10.4 \mathrm{eV}$ is not accessible due to light absorption in water. The second abscissa gives the detector settings for some typical $\xi$ values.

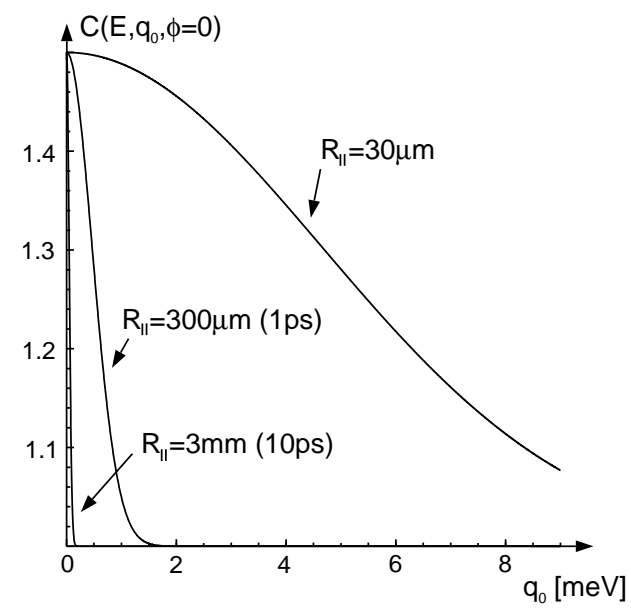

FIG. 2. The correlation function $C\left(E=3 \mathrm{eV}, q_{0}, \phi=0\right)$ as a function of the energy difference $q_{0}$, for various values of $R_{\perp}$. One sees that pulse lengths above 1 ps require an energy resolution well below $1 \mathrm{meV}$. 


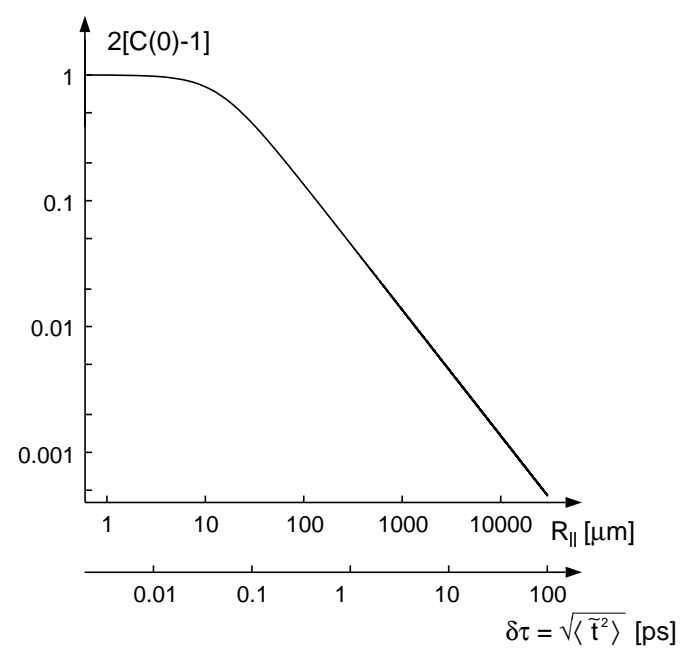

FIG. 3. Effective intercept as a function of the flash duration $R_{\|} \approx c \sqrt{\left\langle\tilde{t}^{2}\right\rangle}$ assuming a filter band width $\delta \lambda=1 \mathrm{~nm}$ at $\lambda=413 \mathrm{~nm}$. 